\title{
Lattice Monte Carlo Simulations in Search of Zeolite Analogs - Effects of Structure Directing Agents
}

\author{
Mohammad Navaid Khan, ${ }^{\dagger}$ Scott M. Auerbach, ${ }^{*, \dagger}$ and Peter A. Monson ${ }^{* \dagger}$ \\ Department of Chemical Engineering, University of Massachusetts, Amherst, and \\ Department of Chemistry, University of Massachusetts, Amherst \\ E-mail: auerbach@chem.umass.edu; monson@ecs.umass.edu
}

In our simulations we considered a bcc lattice with $8 \times 8 \times 8$ unit cells. It should be noted that we have studied systems with $16 \times 16 \times 16$ unit cells as well, however we were unable to obtain ordered microporous materials in PTMC simulations. Falcioni et al. [Marco Falcioni and Michael W. Deem, The Journal of Chemical Physics, 110.3 (1999), 1754-1766] predicted that the total number of PTMC temperature grid points increase as order $\sqrt{N}$ with increase in system size, where $N$ is the system size. Moreover, Katzgarber et al. proposed an optimized scheme for PTMC simulations, where they predicted that the average round trip time for a configuration increases linearly with system size[Katzgraber, H. G., Trebst, S., Huse, D. A., and Troyer, M. (2006), Journal of Statistical Mechanics: Theory and Experiment, 03, P03018]. The above studies indicate that to study a larger system size, a large number of PTMC simulations are required and a longer computational time is needed. We would address this issue in our future publication.

Figure S1 to fig. S5 show 3D connected materials, and fig. S6 to fig. S15 show 2D

\footnotetext{
${ }^{*}$ To whom correspondence should be addressed

${ }^{\dagger}$ Department of Chemical Engineering, University of Massachusetts, Amherst

${ }_{\ddagger}^{\ddagger}$ Department of Chemistry, University of Massachusetts, Amherst
} 
materials observed in our simulations. All structures shown in the text are $2 \times 2 \times 2$ periodic image of an $8 \times 8 \times 8$ bcc lattice.

\section{$3 \mathrm{D}$ materials}

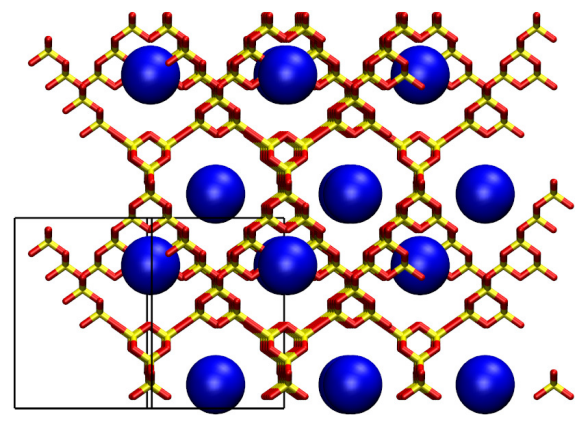

(a)

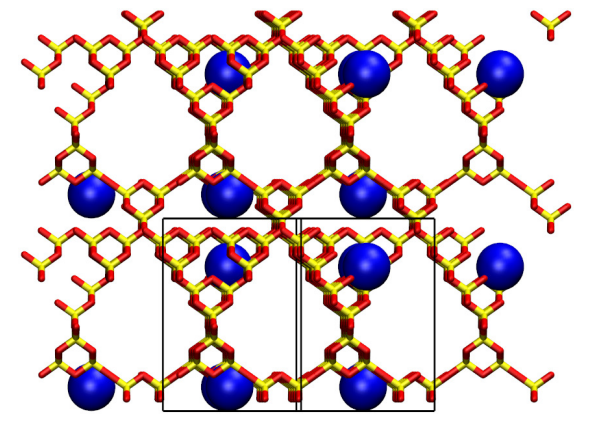

(b)

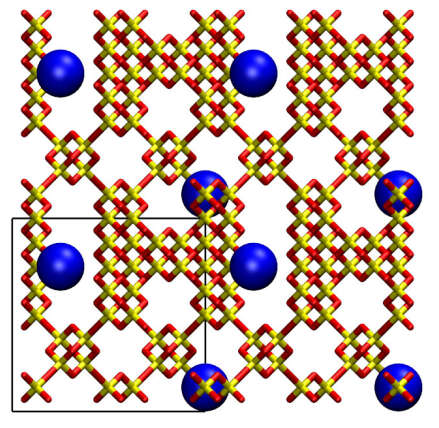

(c)

Figure S1: 3D material with $40 \mathrm{~T}(\mathrm{OH})_{4}, 2$ medium SDAs $\left(D_{S D A}=6.4 \AA\right), \varepsilon_{O-S D A}=$ $0.2 \varepsilon_{T(\mathrm{OH})_{4}-\mathrm{T}(\mathrm{OH})_{4}}$, and $\mathrm{T}^{*}=0.168$. (a) plane $\langle\overline{101}\rangle$; (b) plane $\langle 101\rangle$; (c) plane $\langle 101\rangle$.

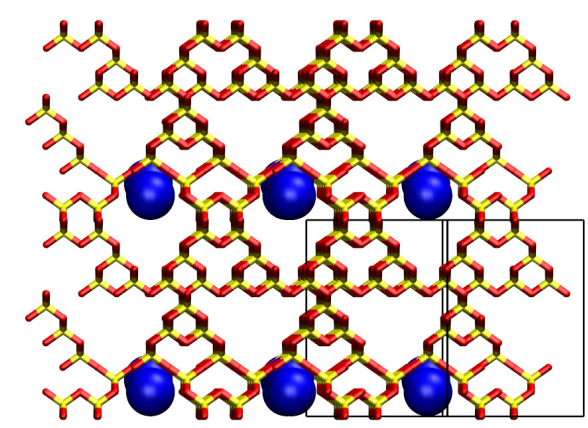

(a)

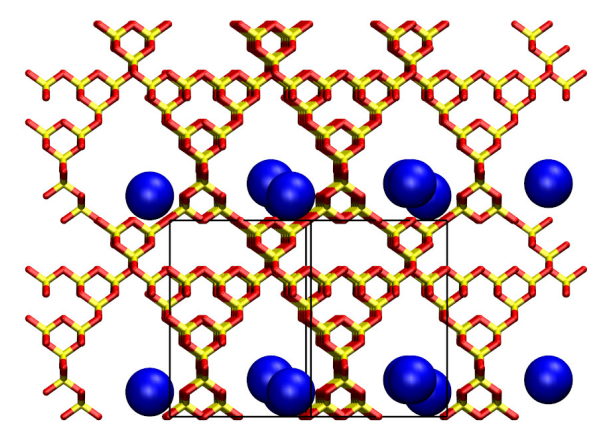

(b)

Figure S2: 3D material with $32 \mathrm{~T}(\mathrm{OH})_{4}, 2$ medium SDAs $\left(D_{S D A}=6.4 \AA\right), \varepsilon_{O-S D A}=$ $0.2 \varepsilon_{T(\mathrm{OH})_{4}-\mathrm{T}(\mathrm{OH})_{4}}$, and $\mathrm{T}^{*}=0.258$. (a) plane $\langle 110\rangle$; (b) plane $\langle\overline{1} 01\rangle$. 


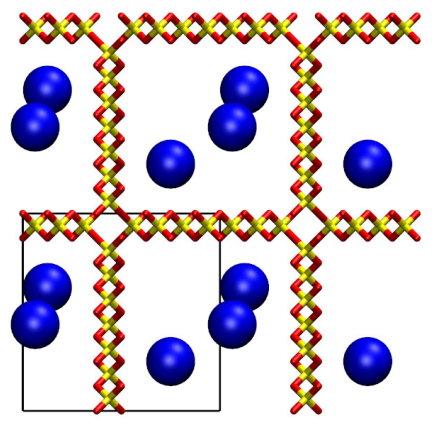

(a)



(b)

Figure S3: 3D material with $56 \mathrm{~T}(\mathrm{OH})_{4}, 3$ medium SDAs $\left(D_{S D A}=6.4 \AA\right), \varepsilon_{O-S D A}=$ $0.2 \varepsilon_{T(\mathrm{OH})_{4}-T(\mathrm{OH})_{4}}$, and $\mathrm{T}^{*}=0.15$. (a) plane $\langle 001\rangle$; (b) plane $\langle 100\rangle$.



(a)

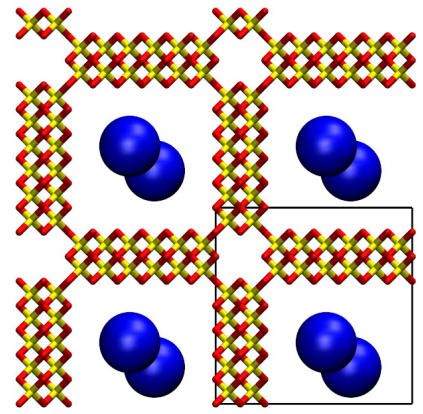

(b)

Figure S4: $3 \mathrm{D}$ material with $48 \mathrm{~T}(\mathrm{OH})_{4}, 2$ large SDAs $\left(D_{S D A}=10.4 \AA\right), \varepsilon_{O-S D A}=$ $0.1 \varepsilon_{T(\mathrm{OH})_{4}-T(\mathrm{OH})_{4}}$, and $\mathrm{T}^{*}=0.156$. (a) plane $\langle 001\rangle$; (b) plane $\langle 100\rangle$.

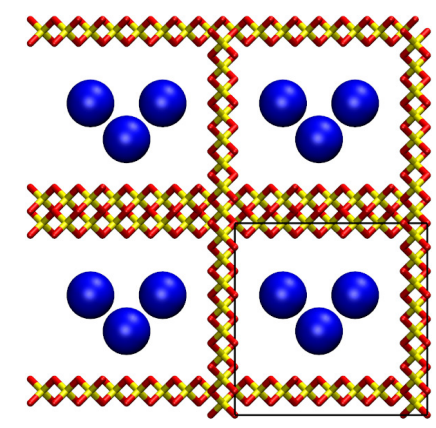

(a)

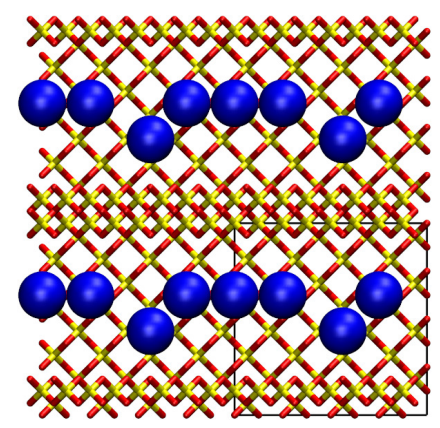

(b)

Figure S5: 3D material with $64 \mathrm{~T}(\mathrm{OH})_{4}, 4$ medium SDAs $\left(D_{S D A}=6.4 \AA\right), \varepsilon_{O-S D A}=$ $0.2 \varepsilon_{T(\mathrm{OH})_{4}-T(\mathrm{OH})_{4}}$, and $\mathrm{T}^{*}=0.204$. (a) plane $\langle 001\rangle$; (b) plane $\langle 100\rangle$. 
2D Materials

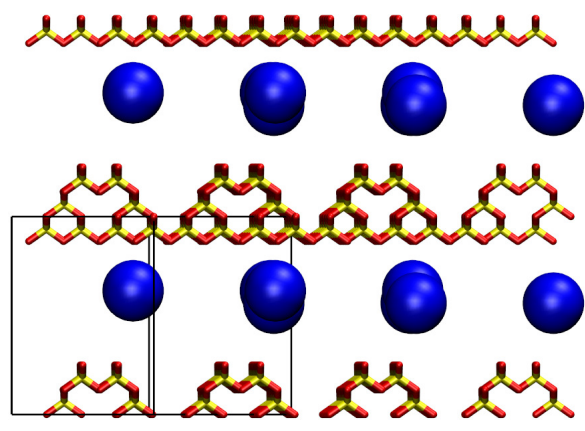

(a)



(b)

Figure S6: $2 \mathrm{D}$ material with $40 \mathrm{~T}(\mathrm{OH})_{4}, 2$ medium SDAs $\left(D_{S D A}=6.4 \AA\right), \varepsilon_{O-S D A}=$ $0.2 \varepsilon_{T(\mathrm{OH})_{4}-T(\mathrm{OH})_{4}}$, and $\mathrm{T}^{*}=0.174$. (a) plane $\langle\overline{1} 01\rangle$; (b) plane $\langle 101\rangle$.

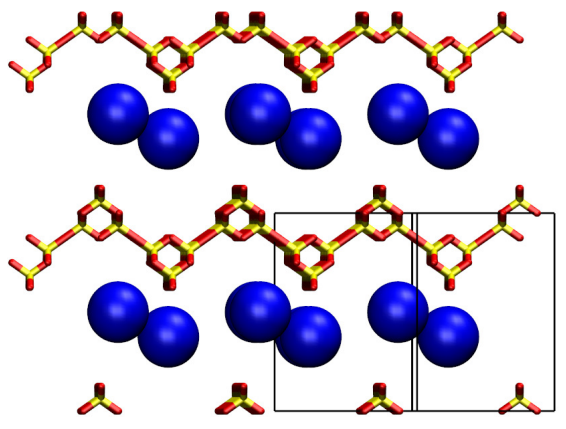

(a)

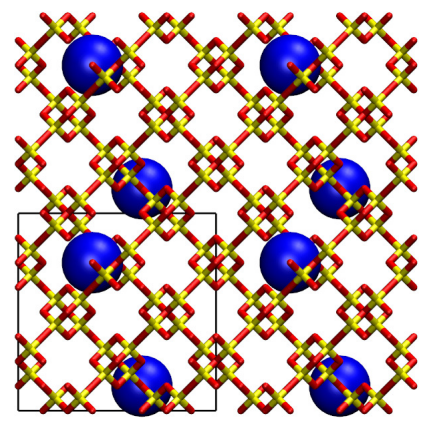

(b)

Figure S7: 2D material with $32 \mathrm{~T}(\mathrm{OH})_{4}, 2$ medium SDAs $\left(D_{S D A}=6.4 \AA\right), \varepsilon_{O-S D A}=$ $0.2 \varepsilon_{T(\mathrm{OH})_{4}-\mathrm{T}(\mathrm{OH})_{4}}$, and $\mathrm{T}^{*}=0.15$. (a) plane $\langle 001\rangle$; (b) plane $\langle\overline{1} 10\rangle$.

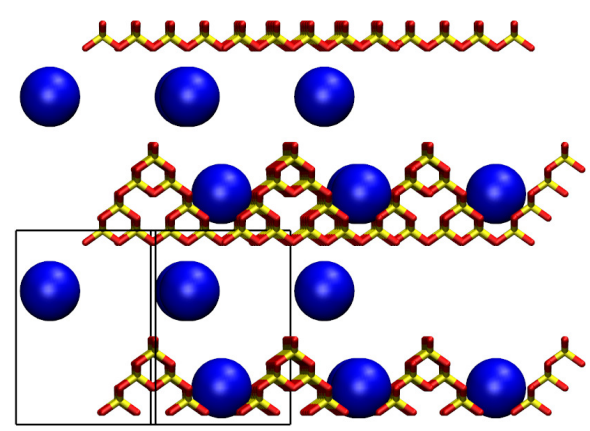

(a)

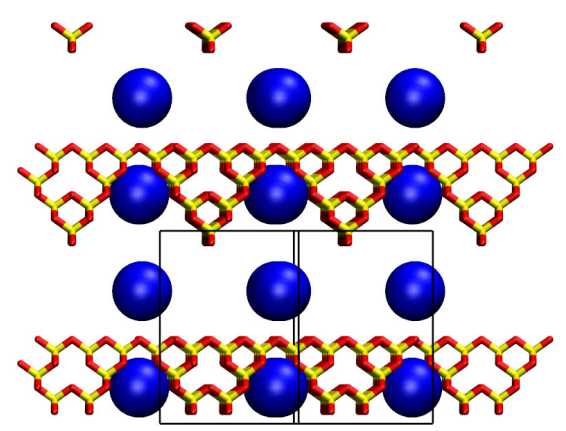

(b)

Figure S8: 2D material with $32 \mathrm{~T}(\mathrm{OH})_{4}, 2$ medium SDAs $\left(D_{S D A}=6.4 \AA\right), \varepsilon_{O-S D A}=$ $0.2 \varepsilon_{T(\mathrm{OH})_{4}-\mathrm{T}(\mathrm{OH})_{4}}$, and $\mathrm{T}^{*}=0.156$. (a) plane $\langle 0 \overline{1} 1\rangle$; (b) plane $\langle 011\rangle$. 


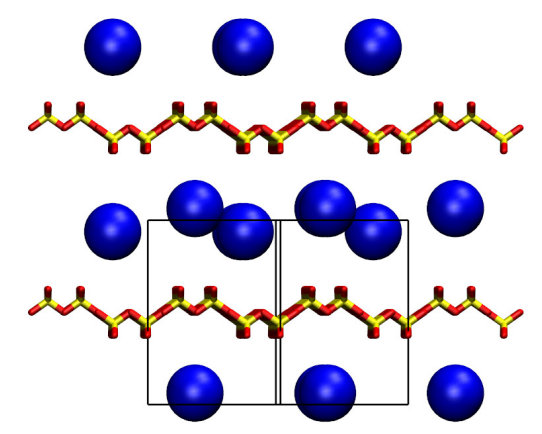

(a)

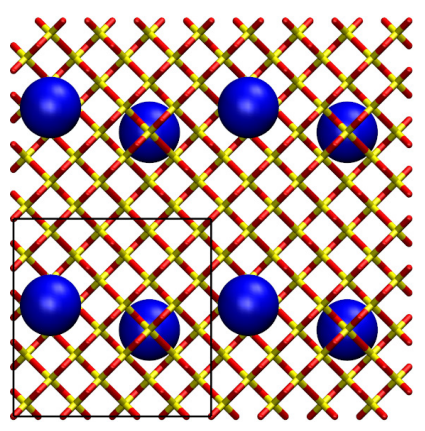

(b)

Figure S9: $2 \mathrm{D}$ material with $32 \mathrm{~T}(\mathrm{OH})_{4}, 2$ medium $\operatorname{SDAs}\left(D_{S D A}=6.4 \AA\right), \varepsilon_{O-S D A}=$ $0.4 \varepsilon_{T(\mathrm{OH})_{4}-T(\mathrm{OH})_{4}}$, and $\mathrm{T}^{*}=0.168$. (a) plane $\langle 101\rangle$; (b) plane $\langle 010\rangle$.

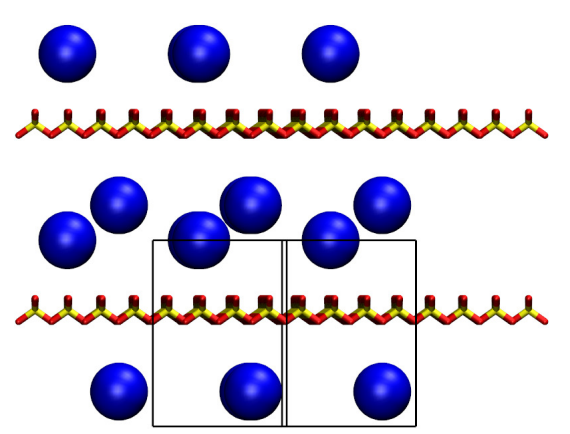

(a)

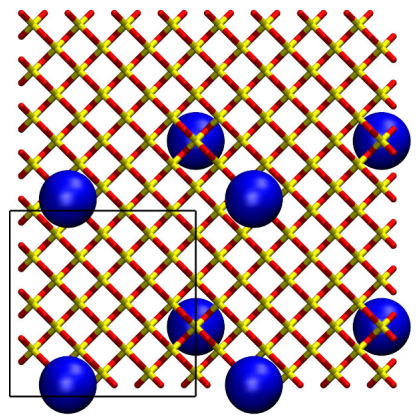

(b)

Figure S10: $2 \mathrm{D}$ material with $32 \mathrm{~T}(\mathrm{OH})_{4}, 2$ medium SDAs $\left(D_{S D A}=6.4 \AA\right), \varepsilon_{O-S D A}=$ $0.4 \varepsilon_{T(\mathrm{OH})_{4}-T(\mathrm{OH})_{4}}$, and $\mathrm{T}^{*}=0.228$. (a) plane $\langle 0 \overline{1} 1\rangle$; (b) plane $\langle 100\rangle$.

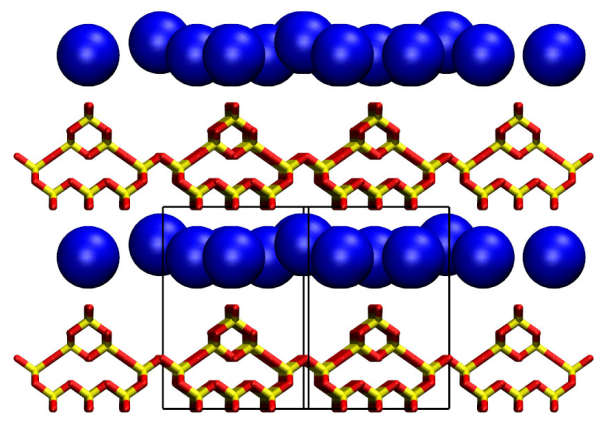

(a)

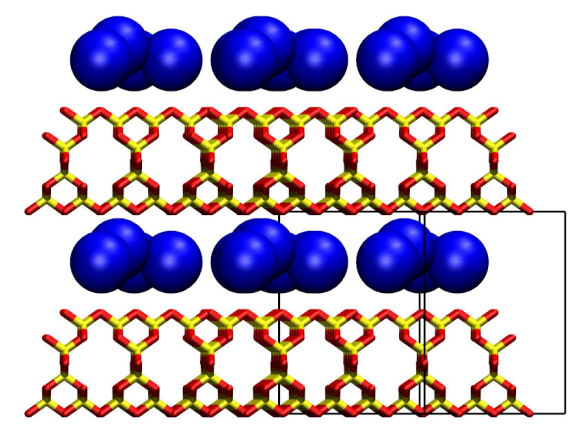

(b)

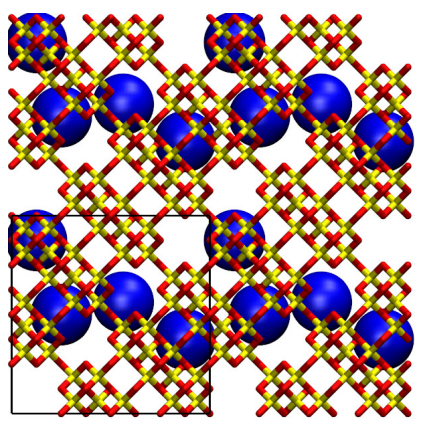

(c)

Figure S11: $2 \mathrm{D}$ material with $48 \mathrm{~T}(\mathrm{OH})_{4}, 4$ medium SDAs $\left(D_{S D A}=6.4 \AA\right), \varepsilon_{O-S D A}=$ $0.2 \varepsilon_{T(\mathrm{OH})_{4}-\mathrm{T}(\mathrm{OH})_{4}}$, and $\mathrm{T}^{*}=0.168$. (a) plane $\langle 011\rangle$; (b) plane $\langle 0 \overline{1} 1\rangle$; (c) plane $\langle 100\rangle$ 


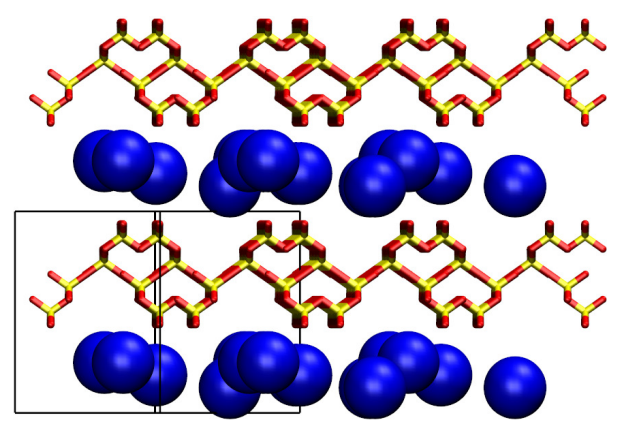

(a)

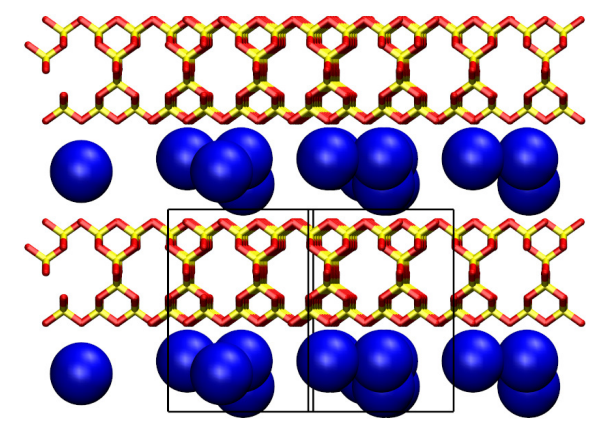

(b)

Figure S12: $2 \mathrm{D}$ material with $48 \mathrm{~T}(\mathrm{OH})_{4}, 4$ medium SDAs $\left(D_{S D A}=6.4 \AA\right), \varepsilon_{O-S D A}=$ $0.2 \varepsilon_{T(\mathrm{OH})_{4}-\mathrm{T}(\mathrm{OH})_{4}}$, and $\mathrm{T}^{*}=0.192$. (a) plane $\langle\overline{1} 10\rangle$; (b) plane $\langle 110\rangle$

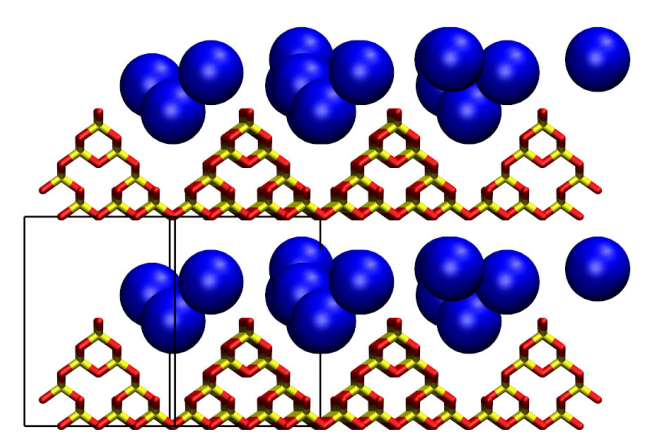

(a)

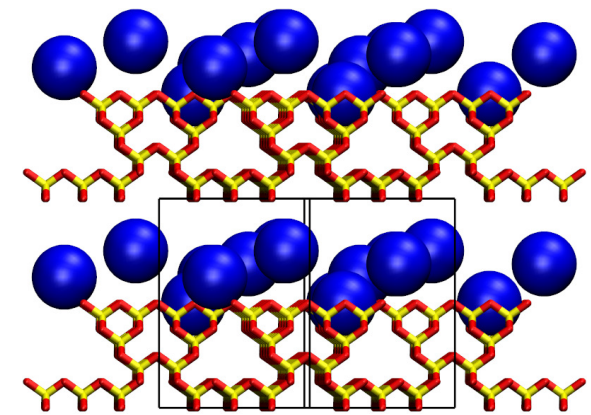

(b)

Figure S13: $2 \mathrm{D}$ material with $48 \mathrm{~T}(\mathrm{OH})_{4}, 4$ medium SDAs $\left(D_{S D A}=6.4 \AA\right), \varepsilon_{O-S D A}=$ $0.2 \varepsilon_{T(\mathrm{OH})_{4}-T(\mathrm{OH})_{4}}$, and $\mathrm{T}^{*}=0.222$. (a) plane $\langle 0 \overline{1} 1\rangle$; (b) plane $\langle 011\rangle$

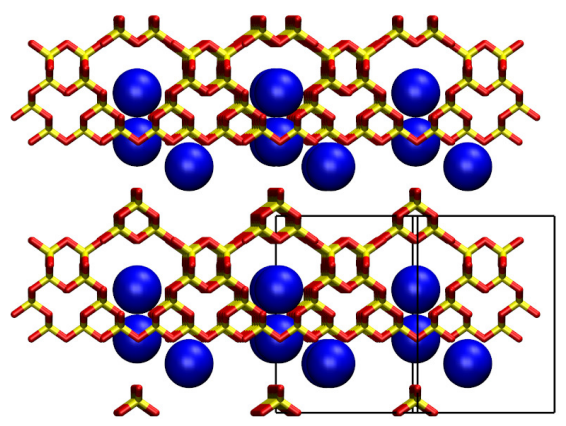

(a)

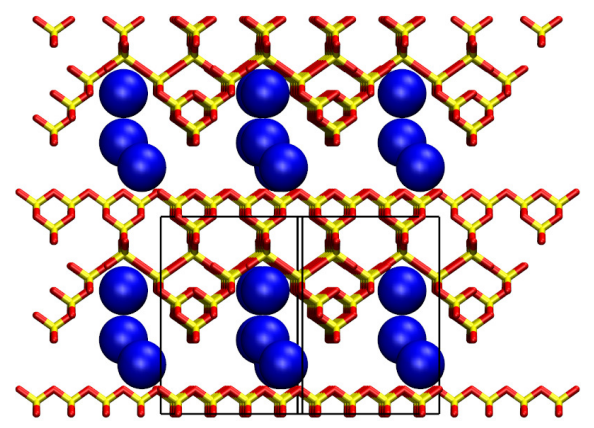

(b)

Figure S14: $2 \mathrm{D}$ material with $48 \mathrm{~T}(\mathrm{OH})_{4}, 3$ medium SDAs $\left(D_{S D A}=6.4 \AA\right), \varepsilon_{O-S D A}=$ $0.2 \varepsilon_{T(\mathrm{OH})_{4}-T(\mathrm{OH})_{4}}$, and $\mathrm{T}^{*}=0.150$. (a) plane $\langle\overline{1} 10\rangle$; (b) plane $\langle 100\rangle$ 


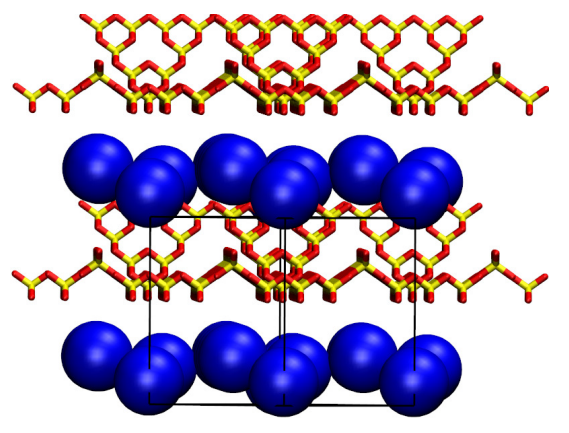

(a)

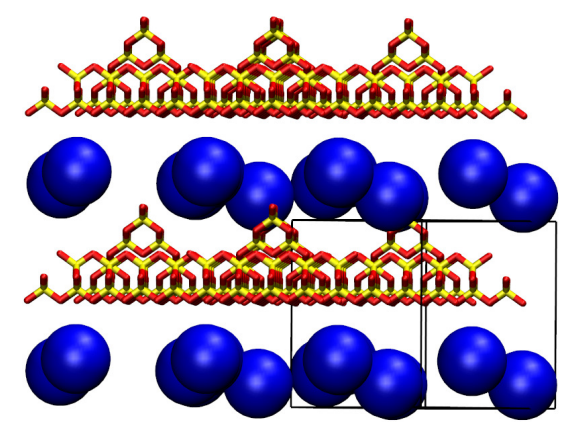

(b)

Figure S15: $2 \mathrm{D}$ material with $48 \mathrm{~T}(\mathrm{OH})_{4}, 4$ medium SDAs $\left(D_{S D A}=10.4 \AA\right), \varepsilon_{O-S D A}=$ $0.2 \varepsilon_{T(\mathrm{OH})_{4}-T(\mathrm{OH})_{4}}$, and $\mathrm{T}^{*}=0.150$. (a) plane $\langle 011\rangle$; (b) plane $\langle 0 \overline{1} 1\rangle$ 